\title{
SYNTAXONOMY AND ECOLOGY OF FOREST VEGETATION IN THE ŠTIAVNICKÉ VRCHY MTS (CENTRAL SLOVAKIA)
}

\author{
Michal SLEZÁK ${ }^{1,2}$, KATARÍNA HEGEDÜŠOVÁ ${ }^{3}$, DUŠAN SENKO ${ }^{3}$ \\ ${ }^{1}$ Faculty of Education, Catholic University \\ Hrabovská cesta 1, SK-034 01 Ružomberok, Slovak Republic \\ 2 Institute of Forest Ecology, Slovak Academy of Sciences \\ Štúrova 2, SK-960 53 Zvolen, Slovak Republic \\ e-mail: slezak.miso@gmail.com \\ ${ }^{3}$ Institute of Botany, Slovak Academy of Sciences \\ Dúbravská cesta 9, SK-845 23 Bratislava, Slovak Republic
}

(Received: April 7, 2010. Accepted: February 2, 2011)

\begin{abstract}
Vegetation of deciduous forests in the Štiavnické vrchy Mts (Central Slovakia) was studied using the standard Zürich-Montpellier approach. The numerical classification and ordination technique were applied to determine the main forest vegetation types and to find the responsible environmental drivers related to their distribution patterns, respectively. The data set including 198 relevés collected by authors in 1997-2009 and 185 relevés excerpted from literature was used to analysis. Numerical classification resulted in delimitation of fourteen vegetation types representing eleven associations with two variants and two communities within the Quercetea roboripetraeae and Querco-Fagetea classes. The major environmental gradients in variation of forest species composition were associated with moisture and nutrient content following the average Ellenberg indicator values. Along the moisture gradient, vegetation types were ordered from subxerophilous oak forests turn mesophilous mixed oak-hornbeam, beech and ravine forests to hygrophilous riparian alder forest. The results confirmed important role of soil nutrients and moisture by determination of forest vegetation in subcontinental part of Central Europe. Special attention was given to the discussion of floristical characteristics, site conditions and syntaxonomy.
\end{abstract}

KEY WORDS: classification, Quercetea robori-petraeae, Querco-Fagetea, numerical methods, Ellenberg indicator values, vegetation survey, Western Carpathians.

\section{INTRODUCTION}

Forest ecosystems are the most important functional component of the Central European landscape, and they are essential for maintaining biological diversity. Spatial distribution and horizontal structure of forest vegetation are simultaneously influenced by a number of ecological and anthropogenic factors and their interactions. The different geology and soil properties, long-term development of vegetation, land-use history and the biogeographical position resulted in a mosaic of plant species and forest communities (e.g. Roo-Zielinska and Solon 1997; Aude and Lawesson 1998; Kolb and Diekmann 2004; Dambrine et al. 2007). Environmental and geographical gradients are therefore the main drivers of variation in floristic composition of temperate deciduous forests. In the last decade, this issue has been discussed in a number of botanical studies, especially those dealing with vegetation classification (e.g. Matuszkiewicz 2000; Willner 2002; Knollová and Chytrý 2004; Boublík et al. 2007; Douda 2008; Towpasz and
Stachurska-Swakoń 2008), evaluation of vegetation-environmental relationships (e.g. Härdtle et al. 2005; Leuschner and Lendzion 2009) and also vegetation dynamics (e.g. Šamonil and Vrška 2008). Because of the increasing threats to forests by a range of human activities, special attention was given to the impact of forest management and air pollution on the herbaceous layer (e.g. von Oheimb and Härdtle 2009). The above-mentioned works have enhanced our understanding of vegetation patterns in these ecological systems. The obtained knowledge should provide initial data for justifying suitable conservation management, integrated ecological landscape planning and also for the establishment of national vegetation surveys or checklists of plant communities (Pott 1992; Borhidi 2003; Matuszkiewicz 2007; Willner and Grabherr 2007; Jarolímek et al. 2008; Onyshchenko 2010). In the territory of Slovakia, studies of these features are relatively limited, and focusing on selected vegetation types, i.e. alder(Šomšák 2000), beech- (Ujházy et al. 2004; Ujházyová 2007) or dry-mesic oak forests (Roleček 2005). 
Štiavnické vrchy Mts are generally considered as a very interesting Slovakian vegetation-landscape type with respect to their location in the transition zone between the Western Carpathian and Pannonian region. This forest vegetation consists of thermophilous as well as submontane (dealpine or praealpine) vascular plants, many of which belong to rare and endangered species (Hlavaček 1985). Deciduous forests are among the most widespread vegetation types of region and their investigation according to the Braun-Blanquet approach has a long history in the Štiavnické vrchy Mts (Mikyška 1929, 1933, 1939; Neuhäusl and Neuhäuslová-Novotná 1964). Although some papers have been recently published (Balkovič 2002; Ciriaková and Hegedüšová-Kučerová 2003; Slezák and Kukla 2009; Slezák and Petrášová 2010), syntaxonomy, ecology and vegetation diversity of these forests have been remained poorly known. Aim of this paper was: (i) to prepare a complex syntaxonomical revision of deciduous forest vegetation in the Štiavnické vrchy Mts as a result of numerical classification, and (ii) to find the major environmental gradients responsible for variation in their floristic composition.

\section{MATERIAL AND METHODS}

\section{Study area}

The research was carried out in the Štiavnické vrchy Mts $\left(48^{\circ} 12^{\prime}-48^{\circ} 35^{\prime} \mathrm{N} ; 18^{\circ} 32^{\prime}-19^{\circ} 05^{\prime} \mathrm{E}\right)$, which represent a clearly defined and homogenous geomorphologic unit in the central part of Slovakia (Fig. 1). They occupy a geographic territory of approximately $800 \mathrm{~km}^{2}$, extending from the colline to the submontane belt. The studied area belongs to the typical volcanic mountains with a quite uniform geological substrate. The bedrock consists mainly of andesites and rhyolites with scattered occurrence of conglomerates and shales. A substantial part of the area has a moderately warm climate. The values of the long-term (1961-1990) mean annual precipitation and air temperature are $771 \mathrm{~mm}$ and $7.7^{\circ} \mathrm{C}$ respectively, the mean air temperature in April-September is $14.0^{\circ} \mathrm{C}$ (climatic station Banská Štiavnica; Slovak Hydrometeorological Institute).

\section{Vegetation sampling and phytosociological analysis}

The base for the study and evaluation of forest communities in the Štiavnické vrchy Mts were the phytosociological data collected during the field research and the ones excerpted from Central Phytosociological Database of Slovakia (CDF, http://ibot.sav.sk/cdf/index.html; Hegedüšová 2007). All the phytosociological relevés were recorded according to the principles of the Zürich-Montpellier approach (Braun-Blanquet 1964), frequently using the modified 9-degree Braun-Banquet's sampling scale (Barkman et al. 1964) and stored in a TURBOVEG database (Hennekens and Schaminée 2001). The CDF relevés with size smaller than $250 \mathrm{~m}^{2}$ have been deleted before the analysis. For numerical analysis, some taxonomically problematic species, which were not distinguished in several relevés, were classified within higher or broadly defined taxa and marked with abbreviation 'agg.': Achillea mille-

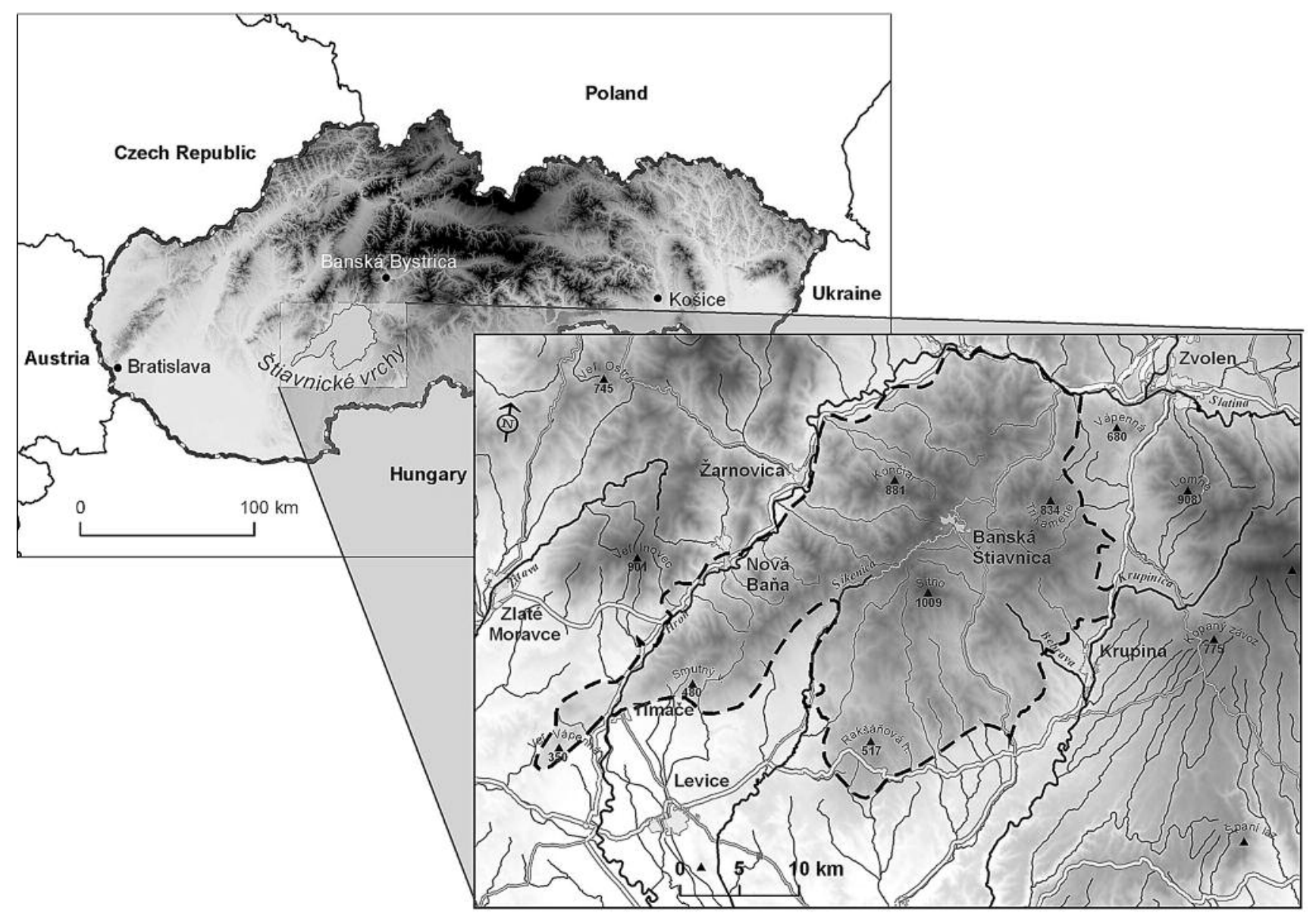

Fig. 1. Location of the study area. 


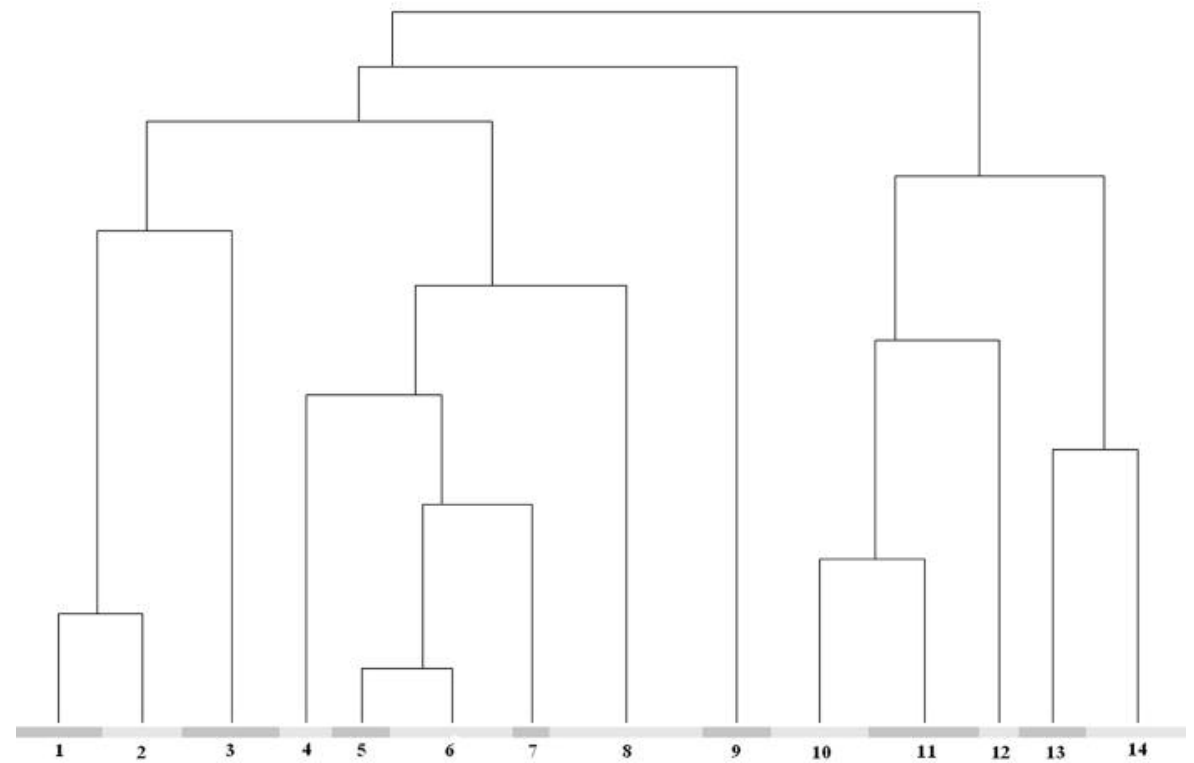

Fig. 2. Dendrogram of numerical classification of the study relevés (for explanation of cluster numbers see Table 1). folium agg. (incl. A. collina, A. millefolium, A. setacea), Festuca ovina agg. (F. ovina, F. valesiaca), Festuca rubra agg. (F. nigrescens, F. rubra), Galium mollugo agg. ( $G$. album subsp. album, G. mollugo, G. pycnotrichum), Poa pratensis agg. (P. angustifolia, $P$. pratensis), Pulmonaria officinalis agg. (P. obscura, P. officinalis), Pyrus communis agg. (P. communis, P. pyraster), Quercus petraea agg. (Q. dalechampii, $Q$. petraea, $Q$. polycarpa), Senecio nemorensis agg. (S. germanicus, $S$. ovatus), Verbascum thapsus agg. (V. densiflorum, V. thapsus), Vicia cracca agg. (V. cracca, $V$. tenuifolia). The resulting set of 383 relevés was compiled; 198 unpublished relevés were taken by the authors in the period 1997-2009 and 185 relevés were obtained from CDF (including all relevés assigned to the classes Quercetea robori-petraeae and Querco-Fagetea by their authors). Numerical classification of the data set was performed by the PC-ORD 5 program (McCune and Mefford 1999) with relative Euclidean distance as a measure of dissimilarity and Ward's linkage method. Species percentage cover was logarithmically transformed. The optimal number of cluster was estimated by the crispness method (Botta-Dukát et al. 2005) using the JUICE software (Tichý 2002). The diagnostic species of the clusters were determined on the basis of the fidelity concept (Chytrý et al. 2002) and frequency (threshold values: $\Phi \geq 0.25$ and frequency $\geq 30$ ) in the JUICE software. The size of all clusters was standardized to an equal size (Tichý and Chytrý 2006). Fisher's exact test $(P<0.001)$ was used to eliminate the fidelity value of species with a non-significant pattern of occurrence.

Detrended correspondence analysis (DCA) from the CANOCO 4.5 for Windows package (ter Braak and Šmilauer 2002) was applied for the ecological interpretation of the main gradients in the studied forest vegetation units. Average Ellenberg indicator values (Ellenberg et al. 1992) and Shannon-Wiener diversity index for relevés were plotted onto a DCA ordination diagram as supplementary variables. The significance of differences in Ellenberg indicator values among the clusters was tested with one-way ANOVA and post-hoc Duncan test. The STATISTICA software (STATSOFT INC. 2006) was used for multiple comparisons and construction of Box and Whisker plots. Altitude and aspect of the vegetation plots were measured by
Garmin GPSmap 60 CSx equipment (WGS-84 system). The environmental characteristics of individual vegetation types (clusters) are in accordance with methodological and result parts of earlier studies (Slezák and Kukla 2009; Slezák and Petrášová 2010). The nomenclature follows Marhold and Hindák (1998) for vascular plants. The names of syntaxa have been unified according to Jarolímek et al. (2008).

\section{RESULTS}

\section{Phytosociological and ecological characteristics of forest communities}

According to the syntaxonomical interpretation and description given below, the fourteen floristically differentiated vegetation units can be assigned to two classes of Quercetea robori-petraeae and Querco-Fagetea (Fig. 2, Tables 1 and 2).

\section{Cluster 1: Carici pilosae-Carpinetum var. typicum}

The Carpathian oak-hornbeam forests with dominant Carex pilosa are developed in the colline belt. The tree layer is mostly uniform; Carpinus betulus is absolutely predominant, Quercus petraea agg. and Fagus sylvatica may be admixed. The species-poor shrub layer has a higher cover degree only in defined spots. Except for the younger individuals of the tree species, Swida sanguinea, Corylus avellana, Acer campestre and Tilia cordata alternate as dominants. In addition to Fragaria vesca, Melica nutans, Pulmonaria officinalis agg. and Viola reichenbachiana, the herb layer is abundant in mesophilous species (e.g. Carex digitata, Galium odoratum, G. schultesii, Lathyrus vernus, Melica uniflora, Stellaria holostea, Symphytum tuberosum, Tithymalus amygdaloides). The participation of thermophilous oak species is generally low.

Cluster 2: Carici pilosae-Carpinetum var. Poa nemoralis

The Poa nemoralis variant is more xeric and it occupies the more open sites. The herb layer is richer in thermophilous and heliophilous vascular plants (Clinopodium vulgare, Cruciata glabra, Cardaminopsis arenosa, Vincetoxicum hirundinaria and Digitalis grandiflora). It has 
TABLE 1. Shortened synoptic table of forest vegetation of the Štiavnické vrchy Mts. Diagnostic species of communities are shaded and ranked by decreasing phi values (phi coefficient $\times 100$, upper indices); only species with phi values $\geq 0.25$ and frequency $\geq 30$ are presented.

\begin{tabular}{|c|c|c|c|c|c|c|c|c|c|c|c|c|c|c|}
\hline Cluster number & 1 & 2 & 3 & 4 & 5 & 6 & 7 & 8 & 9 & 10 & 11 & 12 & 13 & 14 \\
\hline Number of relevés & 29 & 29 & 32 & 17 & 19 & 40 & 12 & 50 & 22 & 32 & 36 & 13 & 22 & 33 \\
\hline Polygonatum multiflorum & $52^{36.8}$ & 8 & $28^{15.6}$ & $v^{\circ}$ & $16^{4.6}$ & 5 & $17^{5.3}$ & 10 & 9 & 3 & 3 & . & . & . \\
\hline Carex digitata & $69^{29.8}$ & $50^{17.4}$ & 12 & $47^{15.5}$ & $37^{8.8}$ & 22 & 17 & $32^{5.6}$ & & 16 & 17 & . & 9 & . \\
\hline Ranunculus lanuginosus & $38^{28.2}$ & 4 & . & . & 5 & 2 & $25^{15.6}$ & 2 & $32^{22.2}$ & 3 & . & . & $14^{4.6}$ & . \\
\hline Pulmonaria officinalis agg. & $86^{28.0}$ & $65^{16.1}$ & $53^{9.0}$ & $53^{8.9}$ & $58^{11.8}$ & 30 & 25 & $54^{9.5}$ & $50^{7.2}$ & 22 & 22 & . & 5 & . \\
\hline Melica nutans & $52^{27.0}$ & $31^{11.1}$ & 3 & $29^{10.1}$ & $37^{15.7}$ & 10 & 8 & 12 & . & 16 & 8 & . & $18^{1.6}$ & . \\
\hline Tilia cordata $\left(\mathrm{E}_{2}\right)$ & $38^{26.2}$ & $15^{5.2}$ & . & $35^{23.8}$ & . & 5 & . & 6 & . & 3 & 3 & . & $32^{20.5}$ & . \\
\hline Ajuga reptans & $79^{26.0}$ & $54^{11.2}$ & 34 & $53^{10.7}$ & $37^{1.3}$ & 35 & $50^{8.9}$ & $46^{6.6}$ & 18 & $53^{10.8}$ & 3 & 8 & 9 & 6 \\
\hline Heracleum sphondylium & $31^{25.6}$ & $12^{4.7}$ & $9^{2.3}$ & . & $11^{3.6}$ & 5 & . & . & $23^{16.7}$ & 3 & 3 & . & 5 & . \\
\hline Viola reichenbachiana & $97^{25.2}$ & $81^{16.4}$ & $78^{14.9}$ & $94^{23.8}$ & $74^{12.5}$ & $78^{14.6}$ & 42 & $88^{20.4}$ & 18 & 6 & 36 & . & 23 & 3 \\
\hline Melica uniflora & $52^{9.4}$ & 19 & $100^{37.4}$ & 18 & $53^{9.9}$ & 20 & 17 & $38^{1.4}$ & 5 & $72^{21.1}$ & $67^{18.1}$ & 15 & 5 & 18 \\
\hline Moehringia trinervia & $17^{5.5}$ & $19^{7.3}$ & $47^{31.8}$ & 12 & 5 & 5 & . & 10 & 9 & 3 & 11 & . & 9 & 6 \\
\hline Quercus robur $\left(\mathrm{E}_{3}\right)$ & . & . & . & $35^{48.9}$ & . & . & . & . & . & 3 & . & . & $9^{8.7}$ & . \\
\hline Corylus avellana $\left(\mathrm{E}_{2}\right)$ & 24 & 19 & 12 & $82^{36.5}$ & 26 & 18 & 8 & 20 & $55^{18.7}$ & 16 & 17 & . & $50^{15.8}$ & 6 \\
\hline Lonicera xylosteum $\left(\mathrm{E}_{2}\right)$ & . & 4 & . & $35^{36.4}$ & 5 & 5 & $8^{3.5}$ & 2 & 5 & . & . & . & $9^{4.4}$ & 3 \\
\hline Picea abies $\left(\mathrm{E}_{3}\right)$ & 7 & 4 & . & $41^{34.6}$ & $11^{2.8}$ & 2 & 8 & $10^{2.3}$ & 5 & . & . & . & $18^{10.8}$ & 3 \\
\hline Carex sylvatica & $34^{20.9}$ & 8 & . & $47^{32.1}$ & $16^{4.3}$ & $22^{10.3}$ & . & 12 & $14^{2.4}$ & . & . & . & . & . \\
\hline Rubus hirtus agg. & $76^{17.6}$ & $46^{1.0}$ & 44 & $100^{31.1}$ & $58^{7.6}$ & $68^{13.0}$ & 8 & $66^{12.1}$ & 18 & 44 & 19 & 23 & 32 & 18 \\
\hline Dryopteris carthusiana & $17^{1.8}$ & . & 3 & $53^{29.6}$ & $21^{4.8}$ & $30^{11.7}$ & 8 & 16 & $45^{23.7}$ & 3 & 3 & . & 9 & . \\
\hline Galium odoratum & $66^{10.3}$ & 46 & $78^{17.3}$ & $100^{29.4}$ & $79^{17.7}$ & 48 & $50^{1.7}$ & $88^{22.8}$ & 18 & 25 & 31 & . & 18 & 12 \\
\hline Dryopteris filix-mas & $76^{13.9}$ & 23 & 44 & $100^{27.3}$ & $84^{18.5}$ & $78^{14.8}$ & $75^{13.4}$ & $74^{12.9}$ & 36 & 3 & 28 & . & $73^{12.1}$ & 18 \\
\hline Fragaria vesca & $69^{17.4}$ & $73^{19.7}$ & $41^{1.2}$ & $82^{25.0}$ & 11 & 22 & $42^{1.8}$ & 36 & 5 & $41^{1.2}$ & $58^{11.3}$ & . & 32 & 27 \\
\hline Acer pseudoplatanus $\left(\mathrm{E}_{3}\right)$ & . & . & 6 & 6 & $58^{51.1}$ & $10^{2.1}$ & $17^{8.9}$ & 6 & 5 & . & . & . & 5 & . \\
\hline Fraxinus excelsior $\left(\mathrm{E}_{3}\right)$ & 3 & . & $12^{7.6}$ & . & $42^{42.1}$ & $18^{13.4}$ & . & 4 & 5 & . & . & . & . & . \\
\hline Mercurialis perennis & $34^{7.8}$ & 12 & $44^{13.9}$ & 18 & $74^{33.7}$ & $35^{8.1}$ & $42^{12.5}$ & $32^{6.1}$ & 9 & . & 19 & . & . & . \\
\hline Ribes uva-crispa & $17^{3.7}$ & 4 & 12 & $18^{4.1}$ & $53^{33.2}$ & $18^{3.9}$ & 8 & 10 & 5 & 6 & 8 & . & 14 & 6 \\
\hline Acer platanoides $\left(\mathrm{E}_{3}\right)$ & . & 8 & $28^{18.6}$ & . & $42^{32.1}$ & 8 & 8 & 6 & 5 & . & $14^{4.8}$ & . & 5 & 3 \\
\hline Actaea spicata & $17^{7.4}$ & 4 & . & 6 & $21^{11.0}$ & $40^{29.0}$ & $25^{14.8}$ & 10 & . & . & . & . & 9 & . \\
\hline Chamerion angustifolium & . & . & . & . & . & . & $42^{60.7}$ & . & . & . & . & . & . & 3 \\
\hline Primula elatior & $10^{7.2}$ & 4 & . & $6^{1.4}$ & 5 & . & $42^{47.9}$ & . & . & . & . & . & . & . \\
\hline Stellaria media & $21^{11.2}$ & . & . & $12^{2.6}$ & . & . & $50^{39.6}$ & . & $27^{17.6}$ & 9 & . & 8 & . & . \\
\hline Cardamine impatiens & 3 & . & 3 & . & $5^{1.0}$ & . & $33^{38.4}$ & 2 & $14^{12.1}$ & . & 3 & . & . & . \\
\hline Oxalis acetosella & $48^{15.3}$ & 4 & . & $59^{22.1}$ & 21 & $30^{3.5}$ & $83^{37.9}$ & $30^{3.5}$ & $45^{13.5}$ & . & . & . & 23 & . \\
\hline Prenanthes purpurea & . & . & . & $12^{9.4}$ & 5 & $12^{10.4}$ & $33^{37.9}$ & 2 & & . & . & . & . & . \\
\hline Acer pseudoplatanus $\left(\mathrm{E}_{2}\right)$ & . & . & 3 & . & $11^{6.9}$ & $10^{6.2}$ & $33^{35.7}$ & 2 & $9^{5.1}$ & . & . & . & . & 3 \\
\hline Senecio nemorensis agg. & $28^{1.5}$ & $31^{3.5}$ & 12 & $47^{13.9}$ & $58^{20.9}$ & $45^{12.6}$ & $75^{31.8}$ & $30^{3.0}$ & . & . & 14 & . & 14 & . \\
\hline Abies alba $\left(\mathrm{E}_{2}\right)$ & 3 & 4 & 3 & $18^{9.8}$ & . & 2 & $33^{25.7}$ & 4 & 5 & . & 6 & . & $32^{24.2}$ & 3 \\
\hline Alnus glutinosa $\left(\mathrm{E}_{3}\right)$ & . & . & . & $\cdot$ & . & . & . & . & $100^{100}$ & . & . & . & . & . \\
\hline Stellaria nemorum & . & . & . & . & . & . & . & . & $64^{78.7}$ & . & . & . & . & . \\
\hline Aegopodium podagraria & $28^{16.1}$ & . & . & . & 5 & 2 & 8 & 2 & $95^{78.6}$ & . & . & . & . & . \\
\hline Carex remota & . & . & . & $6^{2.1}$ & . & . & . & . & $55^{68.6}$ & . & . & . & . & . \\
\hline Lamium maculatum & . & . & 6 & . & $16^{11.2}$ & . & . & . & $64^{66.6}$ & . & . & . & . & . \\
\hline Festuca gigantea & $21^{8.7}$ & 8 & 3 & 12 & 11 & 10 & . & 4 & $82^{63.2}$ & . & 3 & . & . & . \\
\hline Caltha palustris & . & . & . & . & . & . & . & . & $41^{62.6}$ & . & . & . & . & . \\
\hline Alnus glutinosa $\left(\mathrm{E}_{2}\right)$ & . & . & . & . & . & . & . & . & $41^{62.6}$ & . & . & . & . & . \\
\hline Equisetum arvense & . & . & . & . & . & . & . & . & $41^{62.6}$ & . & . & . & . & . \\
\hline Poa trivialis & . & . & . & . & . & . & . & . & $41^{58.9}$ & . & . & . & $5^{2.0}$ & . \\
\hline Sambucus nigra $\left(\mathrm{E}_{2}\right)$ & . & . & . & 6 & $16^{6.9}$ & $18^{8.6}$ & 8 & 4 & $68^{58.3}$ & . & 3 & . & . & . \\
\hline Cirsium oleraceum & $3^{1.0}$ & . & . & . & . & . & . & . & $36^{55.9}$ & . & . & . & . & . \\
\hline Urtica dioica & 7 & . & . & $29^{6.6}$ & $37^{11.8}$ & $45^{17.5}$ & $50^{20.9}$ & . & $100^{55.7}$ & . & 6 & . & 5 & . \\
\hline Rubus caesius & . & . & . & . & . & 2 & . & 2 & $41^{55.2}$ & . & $6^{2.8}$ & . & . & . \\
\hline Salix fragilis $\left(\mathrm{E}_{3}\right)$ & . & . & . & . & . & . & . & . & $32^{55.0}$ & . & . & . & . & . \\
\hline Geranium phaeum & . & . & . & . & . & . & . & . & $32^{55.0}$ & . & . & . & . & . \\
\hline Lycopus europaeus & . & . & . & . & . & . & . & . & $32^{55.0}$ & . & . & . & . & . \\
\hline Padus avium $\left(\mathrm{E}_{2}\right)$ & . & . & . & . & . & . & . & . & $32^{55.0}$ & . & . & . & . & . \\
\hline Euonymus europaeus $\left(\mathrm{E}_{2}\right)$ & $10^{9.1}$ & 4 & . & . & . & . & . & . & $41^{52.7}$ & . & . & . & . & . \\
\hline Lysimachia vulgaris & . & . & . & . & . & . & . & . & $32^{52.1}$ & $3^{1.1}$ & . & . & . & . \\
\hline Stachys sylvatica & 3 & 15 & 9 & $53^{22.7}$ & 21 & $38^{12.0}$ & 17 & 20 & $95^{52.1}$ & . & 6 & . & 5 & . \\
\hline Impatiens noli-tangere & 17 & 8 & 6 & 12 & 16 & $25^{5.4}$ & $42^{17.5}$ & 14 & $82^{46.8}$ & . & 8 & . & 14 & 3 \\
\hline Glechoma hederacea & $21^{8.0}$ & $23^{10.1}$ & $19^{6.3}$ & . & 11 & 8 & . & 8 & $64^{45.4}$ & . & 8 & . & . & . \\
\hline Milium effusum & $10^{4.0}$ & 4 & . & 6 & $11^{4.2}$ & 2 & $17^{10.9}$ & 4 & $41^{37.7}$ & . & . & . & . & . \\
\hline Acer campestre $\left(\mathrm{E}_{2}\right)$ & $31^{10.3}$ & $35^{12.9}$ & $31^{10.4}$ & 6 & . & . & . & 2 & $68^{37.7}$ & $31^{10.4}$ & $19^{1.7}$ & 15 & . & . \\
\hline Galeobdolon luteum agg. & $28^{9.3}$ & 15 & 6 & $18^{1.6}$ & $42^{20.4}$ & 15 & . & $24^{6.5}$ & $64^{36.9}$ & . & 6 & . & . & . \\
\hline Lysimachia nummularia & $17^{13.4}$ & $12^{6.7}$ & . & . & . & . & . & . & $36^{36.0}$ & $12^{7.8}$ & . & . & 5 & . \\
\hline Galium aparine & $24^{1.9}$ & $27^{3.8}$ & $56^{23.7}$ & . & 21 & 8 & . & 6 & $68^{31.8}$ & 6 & $36^{10.0}$ & 8 & $23^{1.0}$ & 15 \\
\hline Rubus idaeus & 10 & . & 3 & $29^{11.7}$ & 11 & 10 & $33^{14.8}$ & 8 & $50^{27.9}$ & . & 11 & . & $32^{13.6}$ & 6 \\
\hline Festuca heterophylla & . & $15^{13.1}$ & 3 & . & . & . & . & . & . & $44^{49.0}$ & 6 & . & . & 3 \\
\hline
\end{tabular}


TABLE 1. Cont.

\begin{tabular}{|c|c|c|c|c|c|c|c|c|c|c|c|c|c|c|}
\hline Cluster number & 1 & 2 & 3 & 4 & 5 & 6 & 7 & 8 & 9 & 10 & 11 & 12 & 13 & 14 \\
\hline Number of relevés & 29 & 29 & 32 & 17 & 19 & 40 & 12 & 50 & 22 & 32 & 36 & 13 & 22 & 33 \\
\hline Lathyrus niger & $31^{10.3}$ & $31^{10.1}$ & 16 & . & . & 10 & . & 8 & . & $72^{40.5}$ & $22^{3.8}$ & $31^{10.1}$ & 9 & 9 \\
\hline Fragaria moschata & $28^{12.5}$ & $19^{5.5}$ & $28^{12.9}$ & . & . & . & . & . & . & $59^{39.0}$ & $19^{5.7}$ & $15^{2.3}$ & 5 & 3 \\
\hline Pyrethrum corymbosum & & $15^{5.6}$ & $12^{2.9}$ & . & . & . & . & . & . & $50^{38.3}$ & $31^{19.9}$ & 8 & 5 & $12^{2.5}$ \\
\hline Lathyrus vernus & $52^{14.9}$ & $54^{16.3}$ & $50^{13.9}$ & . & 11 & 20 & 8 & 28 & . & $88^{37.1}$ & $53^{15.6}$ & & 9 & 15 \\
\hline Melittis melissophyllum & $34^{12.0}$ & $38^{14.8}$ & 19 & 6 & 5 & 12 & . & 10 & . & $69^{36.7}$ & $25^{5.1}$ & $23^{3.7}$ & 9 & \\
\hline Convallaria majalis & $10^{7.2}$ & $12^{8.7}$ & 3 & . & . & 2 & . & . & . & $31^{34.3}$ & $6^{1.0}$ & . & & 3 \\
\hline Galium schultesii & $69^{14.8}$ & $73^{17.1}$ & $75^{18.2}$ & 12 & 5 & 2 & 33 & 16 & . & $97^{30.5}$ & $78^{19.8}$ & 31 & $50^{4.2}$ & $55^{6.7}$ \\
\hline Hypericum hirsutum & $31^{20.6}$ & . & 9 & 6 & 5 & 2 & $25^{14.9}$ & 4 & . & $41^{29.7}$ & . & 8 & . & \\
\hline Carex montana & $10^{4.6}$ & $19^{14.8}$ & & . & 5 & $8^{1.4}$ & . & 4 & . & $31^{28.5}$ & 3 & $8^{1.6}$ & . & \\
\hline Dactylis polygama & $41^{13.2}$ & $35^{8.6}$ & $56^{23.1}$ & . & . & . & . & 8 & 5 & $62^{27.3}$ & $58^{24.5}$ & 8 & 5 & $27^{3.7}$ \\
\hline Stellaria holostea & $41^{13.0}$ & $31^{5.9}$ & $28^{4.1}$ & . & 16 & . & . & 6 & $32^{6.6}$ & $62^{27.2}$ & $33^{7.6}$ & 8 & 23 & $27^{3.6}$ \\
\hline Crataegus monogyna $\left(\mathrm{E}_{2}\right)$ & 7 & 12 & $16^{2.9}$ & 12 & . & 2 & . & 2 & $27^{12.8}$ & $44^{26.7}$ & 11 & $38^{22.2}$ & . & \\
\hline Rosa canina agg. $\left(\mathrm{E}_{2}\right)$ & 21 & 27 & $53^{17.5}$ & $41^{9.9}$ & 21 & 10 & . & 26 & 14 & & $72^{29.6}$ & 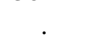 & 14 & $61^{22.2}$ \\
\hline Campanula persicifolia & 24 & $46^{12.5}$ & $34^{5.1}$ & . & 5 & . & 8 & 6 & . & $56^{18.9}$ & $72^{28.9}$ & 23 & $50^{14.9}$ & $42^{10.2}$ \\
\hline Campanula rapunculoides & $48^{16.4}$ & $54^{20.1}$ & $53^{19.6}$ & 6 & 21 & 15 & . & 16 & 5 & 9 & $64^{26.6}$ & 8 & 18 & 9 \\
\hline Festuca rupicola & . & . & . & . & . & . & . & . & . & . & . & $100^{100}$ & . & \\
\hline Seseli osseum & . & . & . & . & . & . & . & . & . & . & . & $85^{89.7}$ & . & 3 \\
\hline Poa compressa & . & . & . & . & . & . & . & . & . & . & . & $77^{86.9}$ & . & \\
\hline Dorycnium pentaphyllum agg. & . & . & . & . & . & . & . & . & . & . & . & $77^{86.9}$ & . & \\
\hline Pilosella bauhinii & . & . & . & . & . & . & . & . & . & . & $11^{2.8}$ & $92^{84.1}$ & 5 & 9 \\
\hline Teucrium chamaedrys & . & . & . & . & . & 2 & . & . & . & 3 & 3 & $77^{74.9}$ & . & $15^{8.6}$ \\
\hline Poa pratensis agg. & . & 4 & . & . & . & . & . & . & . & $31^{18.3}$ & 6 & $92^{72.8}$ & . & $18^{6.6}$ \\
\hline Fragaria viridis & . & . & . & . & . & . & . & . & . & . & . & $54^{72.1}$ & . & \\
\hline Tithymalus cyparissias & . & $15^{2.5}$ & 6 & . & . & . & . & . & . & 12 & $22^{8.2}$ & $92^{67.1}$ & 5 & $21^{7.4}$ \\
\hline Eryngium campestre & . & . & . & . & . & . & . & . & . & . & . & $46^{66.6}$ & . & . \\
\hline Poa pannonica subsp. scabra & . & . & . & . & . & . & . & . & . & . & . & $46^{66.6}$ & . & . \\
\hline Dianthus armeria & . & . & . & . & . & . & . & . & . & $9^{6.5}$ & . & $54^{65.9}$ & . & \\
\hline Trifolium alpestre & . & . & 3 & . & . & . & . & . & . & 3 & $14^{6.9}$ & $69^{65.7}$ & 5 & $9^{1.8}$ \\
\hline Galium verum & $17^{5.0}$ & 8 & . & 6 & . & . & . & . & . & $25^{11.7}$ & 8 & $85^{63.6}$ & 9 & 3 \\
\hline Prunus spinosa $\left(\mathrm{E}_{2}\right)$ & 3 & . & . & . & . & . & . & . & $9^{3.0}$ & $16^{10.4}$ & . & $62^{62.4}$ & . & . \\
\hline Chamaecytisus supinus & . & . & . & . & . & . & . & . & . & $16^{13.6}$ & . & $54^{62.4}$ & . & \\
\hline Viola hirta & . & 4 & . & . & . & . & . & . & . & $28^{23.8}$ & . & $62^{60.9}$ & . & . \\
\hline Cota tinctoria & . & . & . & . & . & . & . & . & . & . & . & $38^{60.6}$ & . & . \\
\hline Rosa gallica & . & . & . & . & . & . & . & . & . & . & . & $38^{60.6}$ & . & . \\
\hline Agrimonia eupatoria & . & . & . & . & . & . & . & . & . & & . & $38^{60.6}$ & . & . \\
\hline Verbascum austriacum & . & 4 & . & . & . & . & . & . & . & $16^{9.7}$ & $8^{1.7}$ & $62^{60.2}$ & . & 6 \\
\hline Trifolium campestre & . & . & . & . & . & . & . & . & . & $9^{7.7}$ & . & $46^{60.0}$ & . & \\
\hline Pimpinella saxifraga agg. & . & . & . & . & . & 2 & . & . & . & . & 6 & $54^{59.5}$ & 5 & $9^{4.5}$ \\
\hline Silene nutans agg. & . & 4 & . & . & . & . & . & . & . & $25^{12.2}$ & $17^{4.9}$ & $77^{58.0}$ & . & $33^{19.6}$ \\
\hline Juniperus communis $\left(\mathrm{E}_{2}\right)$ & . & . & . & . & . & . & . & . & . & 3 & . & $46^{56.6}$ & . & $12^{10.5}$ \\
\hline Pyrus communis agg. $\left(\mathrm{E}_{2}\right)$ & 3 & 4 & . & . & . & . & . & . & 5 & $12^{7.6}$ & 3 & $54^{55.9}$ & . & 3 \\
\hline Trifolium arvense & . & . & . & . & . & . & . & . & . & . & . & $38^{55.8}$ & . & $6^{4.6}$ \\
\hline Agrostis capillaris & . & . & . & . & . & . & . & . & . & $25^{22.0}$ & . & $54^{55.5}$ & . & 6 \\
\hline Achillea millefolium agg. & . & . & . & . & . & . & . & . & . & . & $6^{1.2}$ & $46^{54.5}$ & 5 & $9^{5.8}$ \\
\hline Carex caryophyllea & . & . & . & . & . & . & . & . & . & . & . & $31^{54.0}$ & . & . \\
\hline Inula conyzae & . & . & . & . & . & . & . & . & . & $3^{1.3}$ & . & $31^{51.2}$ & . & \\
\hline Allium senescens subsp. montanum & . & . & . & . & . & . & . & . & . & . & 6 & $46^{51.1}$ & . & $21^{20.0}$ \\
\hline Pseudolysimachion spicatum & . & . & . & . & . & 27 & . & . & . & . & . & $31^{46.6}$ & . & $9^{10.4}$ \\
\hline Festuca ovina agg. & . & . & . & . & . & . & . & . & . & $25^{17.1}$ & $11^{3.0}$ & $54^{46.3}$ & . & $24^{16.3}$ \\
\hline Quercus cerris $\left(\mathrm{E}_{3}\right)$ & 3 & . & 12 & . & . & . & . & . & . & $25^{16.8}$ & $17^{8.4}$ & $54^{45.8}$ & 5 & 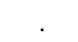 \\
\hline Vicia cassubica & . & . & . & . & . & . & . & . & . & $19^{19.1}$ & $6^{1.4}$ & $38^{45.5}$ & . & \\
\hline Genista tinctoria & 7 & 12 & 6 & . & . & . & . & 2 & . & $53^{23.3}$ & $44^{17.3}$ & $77^{39.9}$ & $23^{2.1}$ & $52^{22.2}$ \\
\hline Hypericum perforatum & . & 12 & 3 & 12 & . & . & . & 4 & . & $50^{23.7}$ & $31^{9.5}$ & $69^{37.7}$ & $23^{3.8}$ & $42^{18.2}$ \\
\hline Vincetoxicum hirundinaria & . & $23^{7.5}$ & 12 & 6 & . & 5 & . & . & . & $25^{9.1}$ & $31^{13.6}$ & $54^{32.3}$ & 9 & $27^{10.9}$ \\
\hline Vaccinium myrtillus & . & . & . & . & . & . & $17^{20.0}$ & . & . & . & . & . & $32^{43.0}$ & \\
\hline Polypodium vulgare & 10 & 12 & 3 & 6 & 11 & 5 & 8 & . & . & . & $28^{11.9}$ & . & $64^{41.2}$ & $39^{21.4}$ \\
\hline Sorbus aucuparia $\left(\mathrm{E}_{2}\right)$ & . & . & . & $24^{20.0}$ & . & 2 & $17^{12.1}$ & . & . & . & . & . & $41^{40.0}$ & 3 \\
\hline Genista pilosa & . & 4 & . & . & . & . & . & . & . & . & $17^{6.1}$ & . & $32^{20.1}$ & $88^{71.9}$ \\
\hline Cardaminopsis arenosa & . & $19^{1.5}$ & 9 & . & . & . & . & 2 & . & . & $50^{24.2}$ & $38^{15.7}$ & $45^{20.8}$ & $76^{43.1}$ \\
\hline Hylotelephium maximum & 14 & 19 & 16 & . & 5 & . & . & . & . & 16 & $47^{18.6}$ & $54^{23.1}$ & $32^{7.9}$ & $82^{42.4}$ \\
\hline Galium mollugo agg. & . & . & 3 & . & . & . & $8^{1.6}$ & 2 & . & 6 & $28^{23.0}$ & . & $9^{2.5}$ & $39^{35.7}$ \\
\hline Digitalis grandiflora & 7 & $19^{2.6}$ & $28^{9.4}$ & . & 5 & . & 8 & 2 & . & $34^{14.1}$ & 4723.9 & 8 & 5 & $58^{31.8}$ \\
\hline Dalanum ladanum & 3 & 4 & $31^{22.4}$ & . & 5 & 5 & . & 4 & . & . & $22^{13.5}$ & 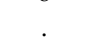 & 9 & $36^{27.5}$ \\
\hline \multicolumn{15}{|c|}{ DS common for several vegetation types } \\
\hline Quercus petraea agg. $\left(\mathrm{E}_{2}\right)$ & 10 & 8 & 6 & & . & & . & & . & $75^{34.3}$ & $39^{10.5}$ & $85^{40.7}$ & $32^{5.9}$ & $67^{28.8}$ \\
\hline Carex pilosa & $100^{41.3}$ & 31 & 25 & $71^{23.7}$ & 21 & $45^{8.4}$ & . & $94^{37.7}$ & 9 & 22 & 8 & . & 9 & 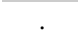 \\
\hline Glechoma hirsuta & $59^{27.2}$ & $31^{7.7}$ & $56^{25.5}$ & 6 & $42^{15.7}$ & 5 & 8 & 14 & $23^{2.1}$ & 9 & $22^{1.8}$ & . & . & \\
\hline
\end{tabular}


TABLE 1. Cont.

\begin{tabular}{|c|c|c|c|c|c|c|c|c|c|c|c|c|c|c|}
\hline Cluster number & 1 & 2 & 3 & 4 & 5 & 6 & 7 & 8 & 9 & 10 & 11 & 12 & 13 & 14 \\
\hline Number of relevés & 29 & 29 & 32 & 17 & 19 & 40 & 12 & 50 & 22 & 32 & 36 & 13 & 22 & 33 \\
\hline Alliaria petiolata & 17 & 15 & $69^{29.0}$ & 6 & $47^{15.1}$ & 25 & . & 18 & $86^{40.5}$ & 6 & $42^{11.4}$ & . & 5 & . \\
\hline Geum urbanum & $31^{7.2}$ & 15 & $59^{26.7}$ & . & 16 & 5 & 8 & 12 & $82^{42.1}$ & $28^{5.2}$ & $28^{5.0}$ & . & . & 3 \\
\hline Impatiens parviflora & $28^{6.1}$ & 15 & $56^{26.4}$ & 12 & 11 & $25^{4.3}$ & . & $22^{2.1}$ & $77^{41.2}$ & . & 14 & . & . & 6 \\
\hline Abies alba $\left(\mathrm{E}_{3}\right)$ & 7 & 4 & . & $76^{35.3}$ & $42^{12.6}$ & $45^{14.5}$ & $100^{50.8}$ & 22 & . & . & 8 & . & 14 & 3 \\
\hline Circaea lutetiana & 14 & 8 & 3 & $53^{26.5}$ & 16 & $38^{15.1}$ & $33^{12.0}$ & $20^{2.2}$ & $55^{27.7}$ & . & . & . & . & . \\
\hline Fagus sylvatica $\left(\mathrm{E}_{3}\right)$ & $72^{10.5}$ & $69^{8.7}$ & 50 & $88^{19.3}$ & $79^{14.1}$ & $100^{25.8}$ & 33 & $100^{25.8}$ & 5 & 6 & 22 & . & $91^{20.8}$ & 33 \\
\hline Viola riviniana & $31^{15.1}$ & 8 & 9 & . & . & . & $50^{30.9}$ & 2 & 9 & $66^{43.8}$ & . & . & 5 & . \\
\hline Hieracium sabaudum & 24 & 23 & 22 & 6 & . & . & . & . & . & $91^{43.5}$ & 22 & $85^{39.6}$ & 18 & $42^{12.1}$ \\
\hline Astragalus glycyphyllos & 21 & 19 & 19 & . & 5 & 2 & . & 6 & . & $72^{36.4}$ & $61^{28.9}$ & $54^{23.8}$ & 5 & 12 \\
\hline Carex muricata agg. & 7 & 12 & 9 & . & 5 & 2 & . & 2 & . & $53^{34.8}$ & $33^{18.0}$ & $46^{28.9}$ & . & . \\
\hline Veronica officinalis & 14 & 15 & 19 & 18 & . & . & . & 18 & . & $72^{33.3}$ & 22 & $77^{36.7}$ & $27^{3.5}$ & $27^{3.5}$ \\
\hline Quercus cerris $\left(\mathrm{E}_{2}\right)$ & . & . & 3 & . & . & . & . & . & . & $34^{33.2}$ & . & $46^{47.0}$ & . & . \\
\hline Ligustrum vulgare $\left(\mathrm{E}_{2}\right)$ & $24^{4.6}$ & $31^{9.4}$ & 6 & . & . & 2 & . & . & 9 & $62^{32.4}$ & $22^{3.2}$ & $85^{48.4}$ & 5 & 3 \\
\hline Clinopodium vulgare & 21 & $42^{11.2}$ & $56^{20.1}$ & . & . & . & . & 4 & . & $75^{32.1}$ & $58^{21.4}$ & $77^{33.4}$ & . & 15 \\
\hline Securigera varia & . & 8 & 6 & . & . & 2 & . & . & . & $22^{9.6}$ & $47^{32.0}$ & $54^{37.9}$ & . & $15^{3.6}$ \\
\hline Galium glaucum & . & . & 3 & . & . & . & . & . & . & 6 & $25^{13.1}$ & $62^{46.1}$ & . & $52^{37.0}$ \\
\hline Linaria genistifolia & . & . & . & . & . & . & . & . & . & . & 6 & $38^{37.2}$ & . & $42^{41.8}$ \\
\hline Steris viscaria & 3 & . & . & . & . & . & . & . & . & 9 & $14^{5.2}$ & $38^{29.4}$ & 5 & $52^{42.3}$ \\
\hline Lembotropis nigricans & 3 & $12^{2.5}$ & . & . & . & . & . & . & . & $19^{9.6}$ & 3 & $38^{28.7}$ & $14^{4.6}$ & $36^{26.7}$ \\
\hline Avenella flexuosa & . & 4 & 3 & 12 & 5 & . & $25^{8.9}$ & . & . & 6 & 14 & 8 & $64^{39.8}$ & $55^{32.5}$ \\
\hline Luzula luzuloides & $52^{3.7}$ & $50^{2.8}$ & 38 & $53^{4.4}$ & 26 & 22 & 33 & $50^{2.8}$ & . & 41 & $64^{10.5}$ & 8 & $100^{30.6}$ & $94^{27.3}$ \\
\hline \multicolumn{15}{|c|}{ Other species with occurrence in more than 12 vegetation types } \\
\hline Poa nemoralis & 48 & $100^{22.0}$ & $94^{18.4}$ & 53 & 37 & 10 & 25 & 28 & 27 & $97^{20.2}$ & $100^{22.0}$ & $100^{22.0}$ & 50 & $91^{16.8}$ \\
\hline Scrophularia nodosa & $31^{9.7}$ & 12 & $28^{7.6}$ & 18 & 16 & $25^{5.3}$ & 17 & $24^{4.6}$ & 5 & $41^{16.6}$ & 6 & 8 & 14 & 6 \\
\hline Mycelis muralis & $66^{8.0}$ & $62^{5.8}$ & 41 & $53^{1.0}$ & $84^{18.3}$ & $62^{6.3}$ & $92^{22.5}$ & $56^{2.7}$ & 18 & $66^{8.0}$ & 47 & 15 & 45 & 9 \\
\hline Geranium robertianum & $52^{3.3}$ & 31 & $75^{16.3}$ & $59^{7.3}$ & $84^{21.4}$ & $55^{5.1}$ & $75^{16.3}$ & 40 & $55^{4.9}$ & 28 & 42 & 15 & 27 & 3 \\
\hline Veronica chamaedrys & 52 & $88^{20.0}$ & $88^{19.5}$ & 6 & . & 2 & 42 & 28 & 5 & $97^{24.7}$ & $92^{21.8}$ & $92^{22.2}$ & $55^{1.2}$ & $88^{19.7}$ \\
\hline Hieracium murorum & 17 & $50^{15.6}$ & 9 & 6 & 5 & 8 & $33^{5.0}$ & 2 & . & $53^{17.6}$ & $42^{10.3}$ & $31^{3.4}$ & $55^{18.5}$ & $45^{12.7}$ \\
\hline Carpinus betulus $\left(\mathrm{E}_{2}\right)$ & $79^{17.5}$ & $81^{18.3}$ & $75^{15.1}$ & $82^{19.2}$ & 32 & 18 & 17 & 38 & 36 & 38 & $78^{16.7}$ & . & 41 & $55^{3.8}$ \\
\hline Fagus sylvatica $\left(\mathrm{E}_{2}\right)$ & $55^{5.0}$ & $58^{6.4}$ & 31 & $82^{20.1}$ & $53^{3.5}$ & $78^{17.4}$ & $58^{6.7}$ & $82^{19.9}$ & 14 & 12 & 28 & . & 649.7 & 33 \\
\hline Carpinus betulus $\left(\mathrm{E}_{3}\right)$ & $100^{22.8}$ & $96^{20.6}$ & $100^{22.8}$ & 9419.5 & $95^{19.8}$ & 55 & . & $66^{3.6}$ & 36 & 28 & $72^{7.1}$ & 8 & 45 & 39 \\
\hline Symphytum tuberosum & $55^{24.8}$ & $35^{10.5}$ & $25^{3.7}$ & . & 16 & 5 & 8 & 12 & $36^{11.7}$ & $44^{16.8}$ & 8 & $23^{2.4}$ & 5 & 3 \\
\hline Quercus petraea agg. $\left(\mathrm{E}_{3}\right)$ & $83^{9.9}$ & $77^{6.5}$ & $97^{18.2}$ & $100^{20.0}$ & 42 & 30 & . & 46 & . & $88^{12.7}$ & $100^{20.0}$ & $100^{20.0}$ & 59 & $100^{20.0}$ \\
\hline Fallopia convolvulus & . & 12 & $50^{21.7}$ & 18 & 11 & 8 & . & 8 & 5 & $22^{1.9}$ & $53^{23.7}$ & $38^{13.6}$ & 9 & $36^{12.1}$ \\
\hline Epilobium montanum & $21^{5.5}$ & $19^{4.3}$ & $16^{1.4}$ & 12 & 11 & $20^{4.9}$ & $25^{9.0}$ & $26^{9.8}$ & 14 & 3 & 6 & . & $23^{7.1}$ & . \\
\hline Brachypodium sylvaticum & $24^{6.8}$ & 15 & 9 & $41^{19.9}$ & 5 & 8 & $33^{13.9}$ & 2 & $23^{5.7}$ & $22^{5.1}$ & 8 & $23^{6.0}$ & . & . \\
\hline Ligustrum vulgare & $45^{15.5}$ & $50^{19.0}$ & $44^{14.8}$ & $29^{5.2}$ & 11 & 8 & . & 8 & 14 & $28^{4.3}$ & $50^{19.0}$ & 15 & . & 3 \\
\hline Dentaria bulbifera & $59^{13.1}$ & 27 & $69^{19.0}$ & 29 & $63^{15.8}$ & $70^{19.7}$ & $42^{3.3}$ & $76^{23.2}$ & 5 & 12 & 22 & . & 23 & 6 \\
\hline
\end{tabular}

Legend: Cluster number 1 - Carici pilosae-Carpinetum var. typicum, 2 - Carici pilosae-Carpinetum var. Poa nemoralis, 3 - Melico uniflorae-Quercetum, 4 - Rubus hirtus agg.-Quercus petraea agg. community, 5 - Aceri-Carpinetum, 6 - Dentario bulbiferae-Fagetum, 7 - Dryopteris filix-mas-Abies alba community, 8 - Cariei pilosae-Fagetum, 9 - Stellario-Alnetum glutinosae, 10 - Festuco heterophyllae-Quercetum, 11 - Poo nemoralis-Quercetum dalechampii, 12 - Poo scabrae-Quercetum, 13 - Luzulo nemorosae-Fagetum, 14 - Luzulo albidae-Quercetum petraeae.

close relation to the Pannonian oak-hornbeam forests on eutrophic soils (Primulo veris-Carpinetum association).

\section{Cluster 3: Melico uniflorae-Quercetum petraeae}

The mixed oak-hornbeam forests are formed by Quercus petraea agg. in the tree layer, less often also by Carpinus betulus and $Q$. cerris. They grow on gentle slopes with warmer and drier microclimate. The shrub layer with low coverage is usually well developed. The species composition of the herb layer changes depending on the site conditions (nutrient availability and soil moisture), but the prevailing species are mostly the diagnostic taxa of the Carpinion betuli alliance and the Fagetalia order (Melica uniflora, Lathyrus vernus, Glechoma hirsuta, Galium schultesii, Alliaria petiolata, Veronica chamaedrys, Geum urbanum, Clinopodium vulgare, Stellaria holostea, Moehringia trinervia, Campanula rapunculoides). This floristically medium-rich community represents the transition types to subxerophilous oak forests.
Cluster 4: Rubus hirtus agg.-Quercus petraea agg. community

The community includes broad-leaved mesophilous forests with dominance of Rubus hirtus agg. Distribution is concentrated on flat areas and gentle slopes. The tree layer is created by Quercus petraea agg., primary accompanied by Carpinus betulus and Fagus sylvatica, locally by Abies alba. The shrub layer is usually represented by the dominant Corylus avellana. The herb layer displays a significant number of species with affinities to mesic habitats (Ajuga reptans, Carex digitata, C. pilosa, C. sylvatica, Fragaria vesca, Geranium robertianum, Mycelis muralis, Oxalis acetosella, Senecio nemorensis agg., Viola reichenbachiana). The ferns enrich the floristic spectrum, but species of Fagion sylvaticae alliance can also be found. A higher frequency of nutrient-demanding species supports a classification of these forests within the Carpinion betuli alliance. 
TABLE 2. Syntaxonomical synopsis of forest vegetation units in the presented area.
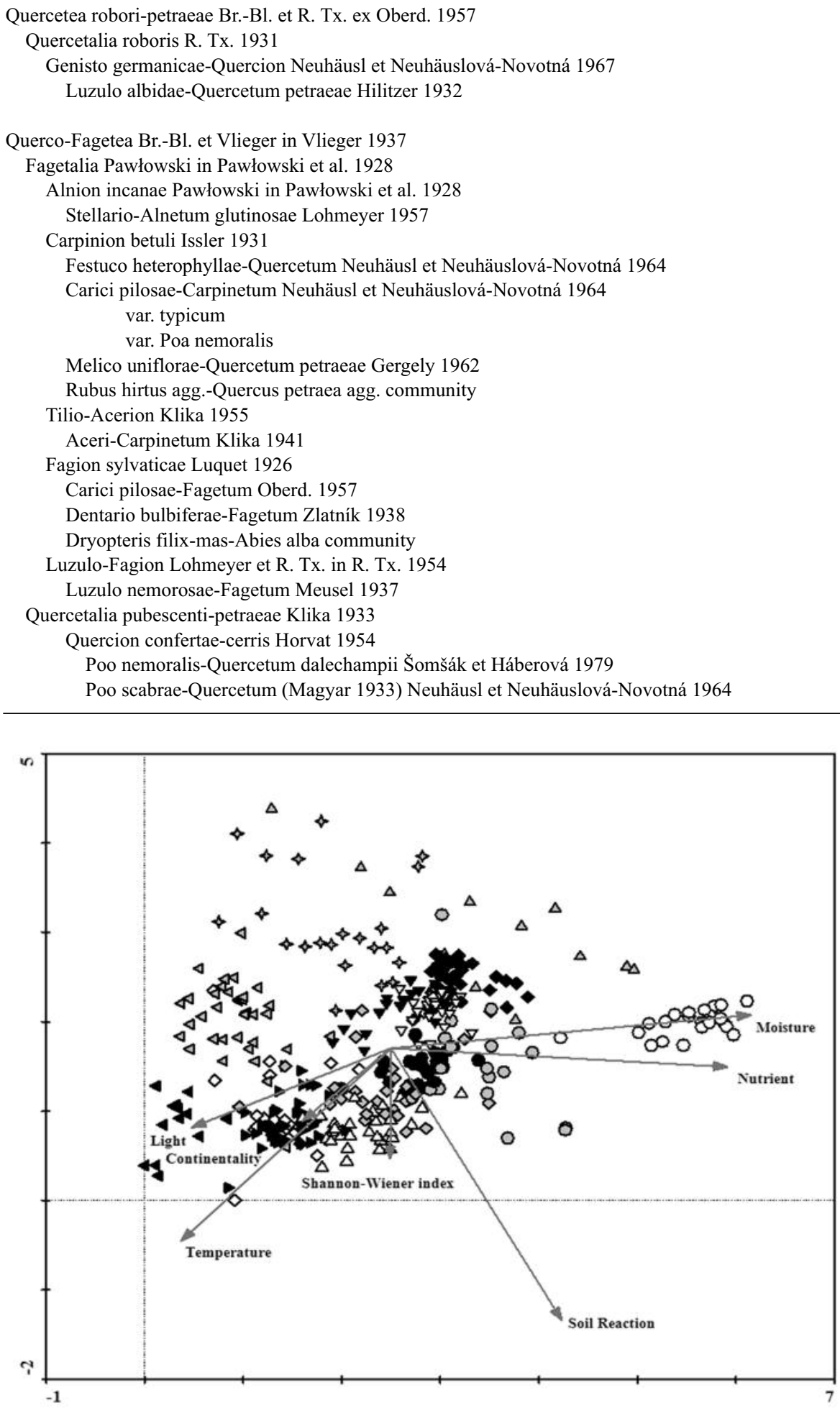

Fig. 3. Detrended correspondence analysis (DCA) ordination diagram of the forest vegetation relevés from the Štiavnické vrchy Mts (length of gradient 6.113; eigenvalues of the first two axes are 0.727 and 0.484 ; total inertia 13.956). Ellenberg indicator values for relevés and Shannon-Wiener index diversity were used as supplementary variables. Correlation coefficients between first two axes and environmental variables: Light $(-0.738$ and $0.040)$, Temperature $(-0.757$ and 0.483$)$, Continentality $(-0.423$ and -0.066$)$, Moisture $(0.954$ and -0.098$)$, Soil reaction $(0.204$ and $0.729)$, Nutrients $(0.920$ and 0.101$)$ and Shannon-Wiener index (-0.152 and 0.021). Black circles (cluster 1), grey diamonds (cluster 2), white up-triangles (cluster 3), black down-triangles (cluster 4), grey circles (cluster 5), black diamonds (cluster 6), grey up-triangles (cluster 7), white down-triangles (cluster 8), white circles (cluster 9), white diamonds (cluster 10), black right-triangles (cluster 11), black left-triangles (cluster 12), stars (cluster 13), grey left-triangles (cluster 14). For the explanation of cluster numbers see Table 1.

\section{Cluster 5: Aceri-Carpinetum}

The thermophilous maple-hornbeam ravine and slopes forests occupying stony slopes or screes are characterised by a considerable species diversity of the tree layer. The mesophilous tree species (Carpinus betulus, Tilia cordata, Acer pseudoplatanus and Fagus sylvatica) prevail, whereas Fraxinus excelsior and Acer platanoides are individually admixed. Due to debris on surface, the shrub layer is weakly developed. A peculiar feature of the herb layer is a high proportion of nitrophilous species indicating favourable humification (e.g. Galeobdolon luteum agg., Mercurialis perennis, Glechoma hirsuta, Alliaria petiolata, Geranium robertianum). Species of the Fagetalia order such as Galium odoratum, Viola reichenbachiana, Dentaria bulbifera, Mycelis muralis and Dryopteris filix-mas are also present. The percentage cover of the grasses Melica uniflora and $M$. nutans is generally low.

\section{Cluster 6: Dentario bulbiferae-Fagetum}

The mesotrophic closed canopy beech communities occurring on gentle slopes with deep siliceous soils are 
species-poor and two-layered forests. The physiognomy of the tree layer is determined by Fagus sylvatica. Other woody species (Carpinus betulus and Abies alba) are less frequent. Except the dominant Dentaria bulbifera, the herb layer is mostly enriched by shade-tolerant species of the Fagetalia order (Actaea spicata, Dryopteris filix-mas and Galium odoratum). Several species including Geranium robertianum and Mycelis muralis indicate mesic and relatively base-rich habitat.

\section{Cluster 7: Dryopteris filix-mas-Abies alba community}

These stands mostly occur on the sites of natural beech forests that occupy the northern slopes. The recent tree species composition is markedly affected by human activities as exploitation of biomass and the following introduction of coniferous trees. The tree layer is built up of Abies alba with scattered occurrence of Fagus sylvatica and Picea abies. The nutrient-demanding species and flora elements of the Fagetalia order (Galium odoratum, Senecio nemorensis agg., Dryopteris filix-mas, Mycelis muralis) are constant components of the species-poor herb layer. The common presence of nitrophilous species such as Cardamine impatiens, Geranium robertianum, Impatiens noli-tangere and Mercurialis perennis indicates the good mineralization of humus. Apart from Prenanthes purpurea, mountain species are absent. Consequently, the floristic composition suggests their assignment to the Fagion sylvaticae alliance.

\section{Cluster 8: Carici pilosae-Fagetum}

Sedge mesophilous beech forests represent the typical vegetation of the Carpathian submontane belt. They are distributed on moderate slopes, their foothills or flat ridges areas; the Carici pilosae-Carpinetum association on warmer sites in colline belt replaced them. Fagus sylvatica is the dominant species of the tree layer; Abies alba, Carpinus betulus and Quercus petraea agg. are sporadically admixed. The overall physiognomy of the herb layer varies according to the canopy openness. It is mostly formed by dominant Carex pilosa which is accompanied by species of the Fagetalia order (e.g. Dryopteris filix-mas, Pulmonaria officinalis agg., Viola reichenbachiana).

\section{Cluster 9: Stellario-Alnetum glutinosae}

The riparian alder forests of nutrient-rich sites growing along small brooks in the colline belt belong to ecologically and floristically well-differentiated communities. The usually three-layered stands are most often created by Alnus glutinosa in the tree layer. The shrub layer is primarily formed by Acer campestre and Sambucus nigra with a frequent admixture of more hygrophilous shrub Euonymus europaeus. The intensity and the character of floods are reflected in their floristic combination. Consequently, the species-rich herb layer encompasses both hygrophytes and nitrophytes (e.g. Aegopodium podagraria, Festuca gigantea, Impatiens noli-tangere, Stellaria nemorum, Stachys sylvatica) together with spring species (Cardamine amara, Carex remota, Chrysosplenium alternifolium). Species of the Calthion alliance including Poa trivialis and Caltha palustris are distinctive.

\section{Cluster 10: Festuco heterophyllae-Quercetum}

The species-rich oak forests with Festuca heterophylla represent marginal community of the Carpinion betuli alliance; they are related on flat ridges in the colline belt. The clear dominance of Quercus petraea agg., locally with presence of $Q$. cerris and Carpinus betulus in the tree layer along with appearance of shrubs (Ligustrum vulgare, Crataegus monogyna, Acer campestre) are distinguished patterns of these stands. The herb layer is dominated by Poa nemoralis in most cases, seldom also by Melica uniflora or Luzula luzuloides. The typical aspect is determined by the mesophilous herbs (e.g. Ajuga reptans, Galium schultesii, Lathyrus vernus, Stellaria holostea, Veronica chamaedrys, Viola riviniana), but acidophilous vascular plants, such as Genista tinctoria, Hieracium murorum, $H$. sabaudum and Veronica officinalis are also constant. The drier and warmer microclimate allows presence of several species of the Quercetalia pubescenti-petraeae order (Astragalus glycyphyllos, Clinopodium vulgare, Lathyrus niger and Melittis melissophyllum) indicating its close relationship to the thermophilous oak communities.

\section{Cluster 11: Poo nemoralis-Quercetum dalechampii}

The moderately opened canopy and floristically-rich oak communities are found commonly in drier habitats with shallow and nutrient-poor soils, mostly on silicates. The stands are characterised by prevalence of Quercus petraea agg. (rarely by $Q$. cerris and Carpinus betulus) in the tree layer and medium-tall grass Poa nemoralis in the herb layer. The development of shrubs is influenced by the intensity of forest management. The constant feature of the understorey vegetation is the occurrence of numerous mesophilous species (e.g. Dactylis polygama, Galium schultesii, Melica uniflora, Cruciata glabra, Stellaria holostea, Campanula rapunculoides, Veronica chamaedrys) together with acidophilous (Genista tinctoria, Hieracium murorum, Luzula luzuloides) and thermophilous oak species (Astragalus glycyphyllos, Cardaminopsis arenosa, Clinopodium vulgare, Digitalis grandiflora, Hylotelephium maximum, Securigera varia, Vincetoxicum hirundinaria). The association is widespread all over the Štiavnické vrchy Mts, mainly in the submontane belt.

\section{Cluster 12: Poo scabrae-Quercetum}

The thermophilous opened canopy oak forests with Poa pannonica subsp. scabra inhabit small stands particularly on south-facing slopes in the colline belt. The tree layer is created by Quercus cerris and $Q$. petraea agg., while Ligustrum vulgare frequently appears in the shrub layer. The heliophytes and acidophytes (e.g. Pilosella bauhinii, Poa angustifolia, P. nemoralis, Veronica officinalis) are typical components of herb layer. The floristic spectrum includes generalists of dry grasslands as well as forest fringes (e.g. Tithymalus cyparissias, Festuca rupicola, F. valesiaca agg., Galium verum, Poa compressa, Teucrium chamaedrys). The abundant group of mesophilous species may enter to the communities on the contact zone with the Carpinion betuli vegetation. The centre of distribution of this species-rich community is in the warmest margins of region.

\section{Cluster 13: Luzulo nemorosae-Fagetum}

The acidophilous beech forests comprise species-poor stands lying on temperate slopes especially in the submontane belt. They are characterized by dominance of Fagus sylvatica with constant admixture of Quercus petraea agg. 
Other woody species such as Carpinus betulus and Abies alba are only accompanying. The occasionally developed shrub layer is made up only with younger individuals of trees. The association is floristically well differentiated within the Fagetalia order through the presence of some oligotrophic elements (Hieracium murorum, Luzula luzuloides, Polypodium vulgare, Vaccinium myrtillus, Veronica officinalis).

\section{Cluster 14: Luzulo albidae-Quercetum petraeae}

The relatively species poor acidophilous oak forests are characterised by the simple vertical structure, the regular occurrence of oligotrophic flora elements and the admixture of thermophilous species. The tree layer is usually composed of Quercus petraea agg., sometimes accompanied by Carpinus betulus. The grassy physiognomy of herb layer results from predominance of Luzula luzuloides, Avenella flexuosa and Calamagrostis arundinacea. Stands are confined to small patches, primarily at the ridge parts and convex spots on the shallow soils.

\section{Ordination}

The scatter plot of DCA, based on individual relevés, shows the distribution of particular vegetation types along the first and second ordination axis. DCA revealed that ten of the fourteen forest vegetation types can be differentiated along the first two ordination axes (Fig. 3). The main compositional gradient along the 1 st axis can be explained by positive correlation of the moisture and nutrient; the negative correlations are ordered along the second axis with respect to soil reaction and temperature. Distribution of relevés in the ordination space shows a continuous ecological transition among the associations. The right part of the scatter-plot is occupied by relevés of the Stellario-Alnetum glutinosae, which occur in the nutrient-rich soils along streams with intense impact of human activities. Duo to their specific ecological conditions such as presence of wet soils together with numerous hygrophilous and nitrophilous species, they are best distinguished within the presented vegetation types. Vegetation plots of the Luzulo albidaeQuercetum petraeae are situated on the opposite end of the ordination diagram. The stands are developed on shallow acidic soils with low nutrient content, and their relation to warmer places of southern slopes with more arid microclimate is marked. Acidophilous beech forests (Luzulo nemorosae-Fagetum) dominate the left upper part of the ordination space, being well differentiated from the Luzulo albidae-Quercetum petraeae, which occurs on the mineral poor soils of the northern slopes in the submontane belt. The thermophilous open dry oak forests (Poo scabraeQuercetum) have the highest requirements of the light conditions. The central part of the scatter-plot is occupied by the Carici pilosae-Carpinetum, partially by Rubus hirtus agg.-Quercus petraea agg. community and the Carici pilosae-Fagetum. Appearance of common oak-hornbeam and beech forest species (e.g. Carex pilosa) is associated with the above-mentioned communities. Similar relationship of these forests to the first two axes is conditioned by their ecological valence. The Melico uniflorae-Quercetum petraeae has a transitional position between mesophilous sedge oak-hornbeam forest and subxerophilous oak forest. This pattern is also reflected in the accepted syntaxonomical classification. The Dryopteris filix-mas-Abies alba community belonging to the Fagion sylvaticae alliance is remarkable different, more oceanic and linked to colder places. The relevés of the Aceri-Carpinetum are positively correlated with nutrient and moisture; these edaphically conditioned ravine forests are typical for sites in lower-altitudinal areas.

Relationships between the defined associations with individual environmental factors are illustrated in figure 4 . The Ellenberg indicator values for moisture are significantly different among the groups of forest stands. The riparian alder forests of Stellario-Alnetum glutinosae (cluster 9) display conspicuous relation on moist and nutrient-rich sites, while Poo scabrae-Quercetum (cluster 12) and Luzulo albidae-Quercetum petraeae (cluster 14) prefer most xeric habitats. Furthermore, the above-mentioned oak forests also exhibit highest light requirements. Luzulo nemorosae-Fagetum (cluster 13) includes forest stands with the most acidic soil reaction.

\section{DISCUSSION AND CONCLUSION}

The deciduous forests represent the prevailing natural vegetation of temperate and submediterranean Europe (Willner et al. 2009). In Slovakia the Querco-Fagetea class is divided into nine alliances containing hygrophilous, mesophilous and (sub)xerophilous forest communities (Jarolímek et al. 2008). There is a high similarity between the national syntaxonomical concept and the structure of forest vegetation classification of Štiavnické vrchy Mts. Thermophilous oak communities related to loess and limestone (Quercion pubescenti-petraeae) or less typically to other basic rock (Quercion petraeae; Aceri tatarici-Quercion) are the only ones missing in the presented area. Acidophilous oak forests are represented with the Luzulo albidae-Quercetum petraeae association (Quercetea roboripetraeae class). Although the dominant Luzula luzuloides is the common oligotrophic forest species, vegetation of the Luzulo albidae-Quercetum petraeae is, in terms of the national classification, relatively rare. A possible explanation lies in (i) the general distribution of acidophilous oak woodlands that are less abundant eastward the Central Europe (Härdtle 2004) and (ii) the transformation of its stands to pine or larch plantations (Moravec 1998). Moreover, the lack of suitable habitats was also emphasized in the geobotanical map of Slovakia (Michalko et al. 1986), where this distinctive community of acidic substrates with an extremely low nutrient supply was not mentioned. Outside the Slovakia this community is often reported under different names. The original description of this community is from the western part of Czech Republic (Hilitzer 1932), but it has been recorded in various European countries (Pallas 1996). The acidophilous oak forests of Genisto germanicae-Quercion alliance are broadly considered to be not sufficiently known vegetation type in the territory of Slovakia. Communities classified within this alliance require syntaxonomical revision in the future. Likewise the previous community, the contemporary phytosociological data of the Stellario-Alnetum glutinosae are becoming more rare. This status is apparently caused by a large-scale reduction of habitats resulting from the direct or indirect influence of human activities, such as stream regulation and drainage. Up to now, these forests with fluctuating 

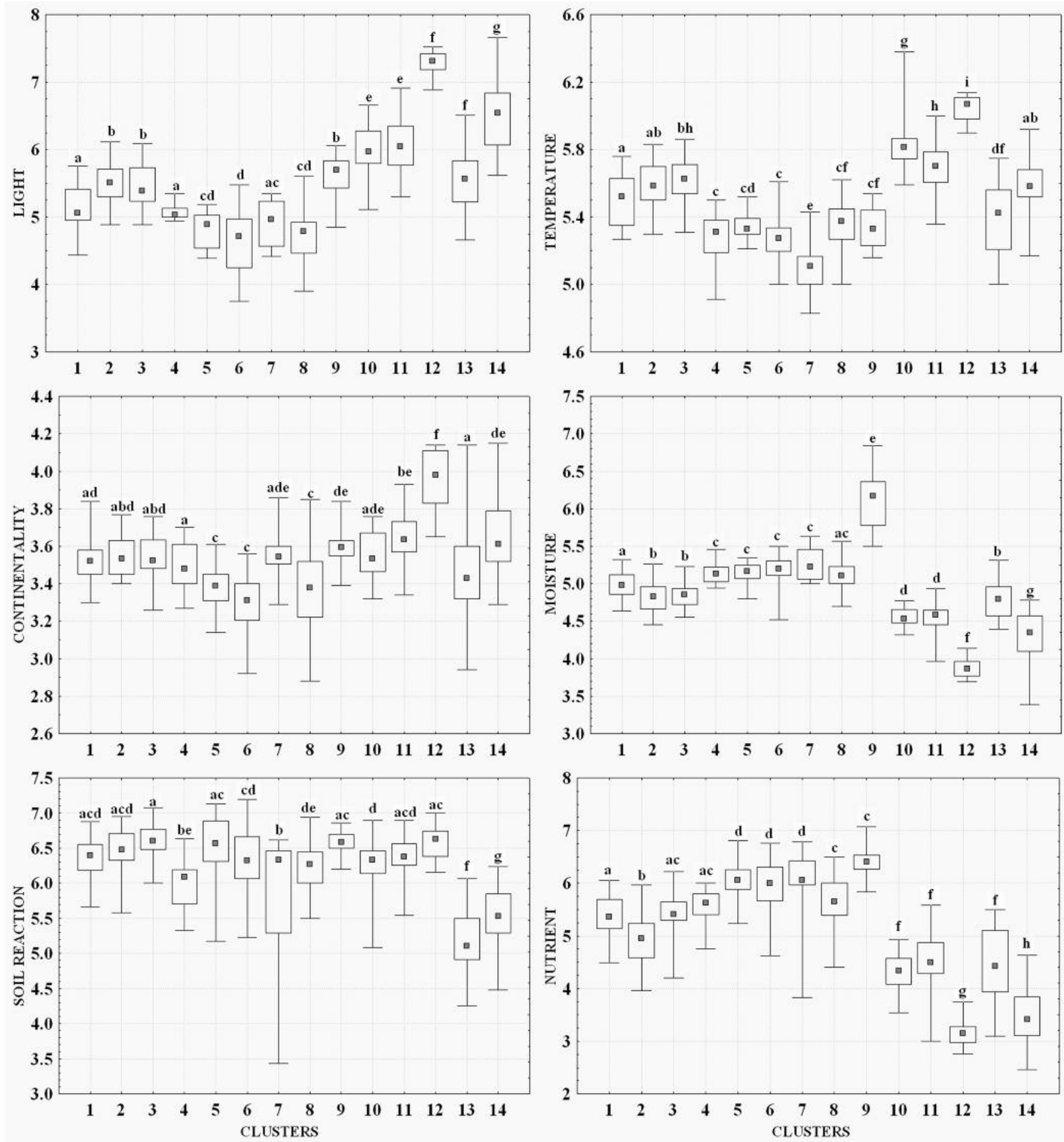

Fig. 4. Box and Whisker plots of the average Ellenberg indicator values for relevés of individual clusters 1-14. The boxes show medians and upper and lower quartiles, while the whiskers indicate the minimum and maximum values. Significant differences among clusters (one-way ANOVA and post-hoc Duncan test, $P<0.05)$ are marked by labels (a-i).

groundwater level were noticed mainly from lowlands and uplands of southern part of Slovakia (Záhorská nížina lowland, Malé Karpaty Mts, Krupinská vrchovina and Cerová vrchovina uplands, Muránska planina plateau), less often from basins and lower mountains, including Volovské vrchy, Vihorlatské vrchy and Zemplínske vrchy Mts (Mikyška 1939; Kárpáti et al. 1963; Miadok 1978).

The oak-hornbeam forests of the Carpinion betuli alliance belong to the important elements of the Carpathian vegetation. They have been intensively discussed because of their geographical and ecological differentiation (Neuhäuslová-Novotná 1964; Neuhäusl 1977; Knollová and Chytrý 2004). Several stands of these mesic broadleaved forests are autochthonous, but many others are developed on natural beech- and partially on oak habitats (Ellenberg 1982). The distribution centre of the Carpinion betuli communities is located in lower-altitudinal areas with subcontinental climate. These conditions are typical for many parts of Slovak volcanic mountains. The most frequently dominating community is the Carici pilosaeCarpinetum that was described as an association for the first time from Štiavnické vrchy Mts by Neuhäusl and Neuhäuslová-Novotná (1964). The geographical range of this association comprises Slovakia, the adjacent parts of 
the Czech Republic (Moravia region), Austria, Hungary, Poland, Ukraine, and Romania (Moravec et al. 2000; Willner and Grabherr 2007; Onyshchenko 2010). Due to the small number of available relevés and their relatively large variability in species composition, it was not possible to distinguish the community Primulo veris-Carpinetum reported by Neuhäusl and Neuhäuslová (1964) from southern foothills of Štiavnické vrchy Mts and occurring also in other parts of the Pannonian region (chiefly uplands of Danube basin). The oak-hornbeam forests of Festuco heterophyllae-Quercetum with transitional character between the Carpinion betuli and Quercion petraeae alliances are less frequent.

There are several studies devoted to floristic differentiation patterns of central European beech forests (Klika 1936; Matuszkiewicz and Matuszkiewicz 1973; Moravec 1985, 1999; Matuszkiewicz 2000; Willner 2002; Boublík et al. 2007; Ujházyová 2007). If the markedly wide ecological amplitude of Fagus sylvatica and its high competitive ability are taken into account (Leuschner et al. 2006), the beech forest vegetation undoubtedly belongs to the most extended group of forest communities in the Western Carpathians. Except the acidophilous beech forests, these vegetation types represent stands of mesotrophic to eutrophic, more humid and cold habitats in comparison with the Carpinion betuli communities. In the studied area they were assigned to two alliances: the forests of the Fagion sylvaticae contain mainly nutrient-demanding species and are developed on mineral-rich soils with mull form of humus, while the forests of the Luzulo-Fagion are distributed on acidic soils with occurrence of typically oligotrophic species. The mesotrophic communities of the Carici pilosae-Fagetum and Dentario bulbiferae-Fagetum are floristically relatively difficult to distinguish from other Central European beech associations. The findings of the present study are in accordance with previous observations (Moravec et al. 2000; Ujházy et al. 2004), that the diagnostic species of other beech forest vegetation types were absent. Both these communities were recorded by numerous phytosociological relevés, and they are generally regarded as the most common and widespread associations of the Eu-Fagenion suballiance in Slovakia. Natural beech forests were strongly exploited in the last centuries and their species composition was partially altered by the introduction of coniferous trees (Härdtle et al. 2005). Stands with prevailing Abies alba in the tree layer (Dryopteris filix-mas-Abies alba community; cluster 7, Table 1) occupy gentle slopes with north aspect at altitudes between 550$900 \mathrm{~m}$. The herb layer is species-poor, elements of mountain flora are absent and acidic-tolerant species are scattered. Additionally to the herbs of nutrient-rich soils (Mercurialis perennis, Geranium robertianum, Cardamine impatiens), the group of beech forest species (Galium odoratum, Senecio nemorensis agg., Dryopteris filix-mas) reflects position of these forests within the Fagion sylvaticae alliance.

The distribution of Fagus sylvatica is primarily limited by longer drought periods, but it is also sensitive to late spring frosts (Bolte et al. 2007). The low soil water availability determinates its occurrence along the moisture gradient. Owing to physiological drought stress, Fagus sylvat$i c a$ is substituted by more drought-tolerant tree species $(Q$. cerris, $Q$. pubescens, $Q$. petraea agg.) in colline belt. As a consequence, drier habitats with stony soils are covered by subxerophilous and thermophilous forest communities. In the case of Štiavnické vrchy Mts, they can be found as part of the canopy-opened oak forests, namely the Poo nemoralis-Quercetum dalechampii and Poo scabrae-Quercetum associations. Within the range of these two communities, the stands of the Poo nemoralis-Quercetum dalechampii are more mesophilous and often concentrated in relatively high-altitudinal areas (400-775 $\mathrm{m}$ a.s.1.). In total, Carpathian subendemic tall grass Poa pannonica subsp. scabra grows on sunny and stony slopes in some types of forest and grassland vegetation. It largely occurs on southern fringes of the Western Carpathians (e.g. Pohronský Inovec, Kremnické vrchy, Štiavnické vrchy, Javorie Mts, Slovenský kras karst), in the northern part of the Hungarian Central Range as well as in Ukraine and Romania (Kliment 1999). For the territory of Slovakia, all available phytosociological relevés of Poo scabrae-Quercetum have been collected only within the Central Slovak volcanic mountains (Krupinská vrchovina upland and Štiavnické vrchy Mts). This peculiar community is surrounded by forests of the Festuco heterophyllae-Quercetum or Quercetum petraeae-cerris associations on mineral-rich and deep soils (Neuhäusl and Neuhäuslová 1964; Roleček 2005).

Vegetation-environmental patterns of deciduous forest are evidently scale dependent across the subcontinental part of Central Europe. Environmental variables connected with different soil and light conditions are generally significant in the determination of forest vegetation distribution at local level, whereas geographical and/or climatic gradient has a decisive role at large-scale (Ellenberg 1982; Knollová and Chytrý 2004). The results of DCA analysis supported that both moisture and soil nutrient are among the most important gradients affecting floristic composition in the study area. Significant influence of soil water supply and available nutrients on species composition of forest vegetation has been already published (e.g. Douda 2008; Leuschner and Lendzion 2009; Slezák and Petrášová 2010).

The mountain units situated on the boundary between the Carpathian and Pannonian region represent regional diversity hotspots of flora elements and plant communities. The present study underscores variability of forest vegetation in the Štiavnické vrchy Mts. Ecological range of recorded vegetation units is expressed with their assignment to the Quercetea robori-petraeae and Querco-Fagetea classes. Floristic differentiation of deciduous forests is simultaneously controlled by soil moisture and nutrient availability.

\section{ACKNOWLEDGEMENTS}

The authors are grateful to J. Kukla for help with field research, R. Hrivnák and J. Kliment for valuable comments to manuscript of this paper. We also thank B. Jarčuška for editing Fig. 4 and P. Zuccarini for language improvement. The research was supported by the Science Grant Agency of the Ministry of Education of the Slovak Republic and Slovak Academy of Sciences (VEGA 2/0068/10, VEGA 2/0034/10, VEGA 2/0059/11) and the Centre of Excellence "Adaptive Forest Ecosystems" funded by the Operational Programme Research and Development financed from the European Regional Development Fund (ITMS: 262201200 006). 


\section{LITERATURE CITED}

AUDE E., LAWESSON J.E. 1998. Vegetation in Danish beech forests: the importance of soil, microclimate and management factors, evaluated by variation partitioning. Plant Ecol. 134: 53-65.

BALKOVIČ J. 2002. Forest vegetation in the north-western part of the Štiavnické vrchy Mts. Phytopedon 2002(1): 17-32.

BARKMAN J.J., DOING H., SEGAL S. 1964. Kritische Bemerkungen und Vorschläge zur quantitativen Vegetationsanalyse. Acta Bot. Neerl. 13: 394-419.

BOLTE A., CZAJKOWSKI T., KOMPA T. 2007. The north-eastern distribution range of European beech - a review. Forestry 80: 413-429.

BORHIDI A. 2003. Magyarország növénytársulásai. Akadémiai Kiadó, Budapest. (in Hungary)

BOTTA-DUKÁT Z., CHYTRÝ M., HÁJKOVÁ P., HAVLOVÁ M. 2005. Vegetation of lowland wet meadows along a climatic continentality gradient in Central Europe. Preslia 77: 89-111.

BOUBLÍK K., PETŘÍK P., SÁDLO J., HÉDL R., WILLNER W., ČERNÝ T., KOLBEK J. 2007. Calcicolous beech forests and related vegetation in the Czech Republic: a comparison of formalized classifications. Preslia 79: 141-161.

BRAUN-BLANQUET J. 1964. Pflanzensoziologie. Grundzüge der Vegetationskunde. Ed. 3. Springer, Wien.

CHYTRÝ M., TICHÝ L., HOLT J., BOTTA-DUKÁT Z. 2002. Determination of diagnostic species with statistical fidelity measures. J. Veg. Sci. 13: 79-90.

CIRIAKOVÁ A., HEGEDÜŠOVÁ-KUČEROVÁ K. 2003. The forest communities of Northwest part of the Štiavnické vrchy Mountains and their ecological characteristics. Ekológia 22: 8-15.

DAMBRINE E., DUPOUEY J.L., LAÜT L., HUMBERT L., THINON M., BEAUFILS T., RICHARD H. 2007. Present forest biodiversity patterns in France related to former Roman agriculture. Ecology 88: 1430-1439.

DOUDA J. 2008. Formalized classification of the vegetation of alder carr and floodplain forests in the Czech Republic. Preslia 80: $199-224$

ELLENBERG H. 1982. Vegetation Mitteleuropas mit den Alpen. Ulmer, Stuttgart.

ELLENBERG H., WEBER H.E., DÜLL R., WIRTH V., WERNER W., PAULISSEN D. 1992. Zeigerwerte von Pflanzen in Mitteleuropa. Scr. Geobot. 18: 1-258.

HÄRDTLE W. 2004. Bodensaure Eichen- und Eichenmischwälder Europas. Tuexenia 24: 57-72.

HÄRDTLE W., VON OHEIMB G., WESTPHAL C. 2005. Relationships between the vegetation and soil conditions in beech and beech-oak forests of northern Germany. Plant Ecol. 177: 113-124.

HEGEDÜŠOVÁ K. 2007. Centrálna databáza fytocenologických zápisov (CDF) na Slovensku. Bull. Slov. Bot. Spoločn. 29: 124-129. (in Slovak with English summary)

HENNEKENS S.M., SCHAMINÉE J.H.J. 2001. TURBOVEG, a comprehensive data base management system for vegetation data. J. Veg. Sci. 12: 589-591.

HILITZER A. 1932. Bory na Horšovotýnsku. Čas. Nár. Mus. 106: 1-12. (in Czech)

HLAVAČEK A. 1985. Flóra CHKO Štiavnické vrchy. ÚŠOP, Bratislava. (in Slovak)

JAROLÍMEK I., ŠIBÍK J., HEGEDÜŠOVÁ K., JANIŠOVÁ M., KLIMENT J., KUČERA P., MÁJEKOVÁ J., MICHÁLKOVÁ D., SADLOŇOVÁ J., ŠIBÍKOVÁ I., ŠKODOVÁ I., UHLÍŘOVÁ J., UJHÁZY K., UJHÁZYOVÁ M., VALACHOVIČ M., ZALIBEROVÁ M. 2008. A list of vegetation units of Slovakia. In: I. Jarolímek, J. Šibík (eds), Diagnostic, constant and dominant species of the higher vegetation units of Slovakia. Veda, Bratislava, pp. 295-329.

KÁRPÁTI V., KÁRPÁTI I., JURKO A. 1963. Bachbegleitende Erlenauen im eukarpatischen und pannonischen Mittelgebirge. Biologia 18(2): 97-120.
KLIKA J. 1936. Das Klimax-Gebiet der Buchenwälder in der Westkarpathen. Beih. Bot. Cbl. 55: 117-157.

KLIMENT J. 1999. Komentovaný prehl’ad vyšších rastlín flóry Slovenska, uvádzaných v literatúre ako endemické taxóny. Bull. Slov. Bot. Spoločn. 21, Suppl. 4: 1-434. (in Slovak with English summary)

KNOLLOVÁ I., CHYTRÝ M. 2004. Oak-hornbeam forests of the Czech Republic: geographical and ecological approaches to vegetation classification. Preslia 76: 291-311.

KOLB A., DIEKMANN M. 2004. Effects of environment, habitat configuration and forest continuity on the distribution of forest plant species. J. Veg. Sci. 15: 199-208.

LEUSCHNER C., MEIER I.C, HERTEL D. 2006. On the niche breadth of Fagus sylvatica: soil nutrient status in 50 Central European beech stands on a broad range of bedrock types. Ann. For. Sci. 63: 355-368.

LEUSCHNER C., LENDZION J. 2009. Air humidity, soil moisture and soil chemistry as determinants of the herb layer composition in European beech forests. J. Veg. Sci. 20: 288-298.

MARHOLD K., HINDÁK F. 1998. Checklist of non-vascular and vascular plants of Slovakia. Veda, Bratislava.

MATUSZKIEWICZ W. 2000. The systematic position of thermophilous beechwoods (Cephalanthero-Fagenion) in Poland. Fragmenta Flor. Geobot. 45(1-2): 393-412.

MATUSZKIEWICZ W. 2007. Przewodnik do oznaczania zbiorowisk roślinnych Polski. Wydawnictwo Naukowe PWN, Warszawa. (in Polish)

MATUSZKIEWICZ W., MATUSZKIEWICZ A. 1973. Przegląd fitosocjologiczny zbiorowisk leśnych Polski. Cz. 1. Lasy bukowe. Phytocoenosis 2: 143-202. (in Polish)

MCCUNE B., MEFFORD M.J. 1999. PC-ORD, Multivariete analysis of ecological data, version 4.0. MjM Software Design, Oregon.

MIADOK D. 1978. Bachbegleitende Erlenbrüche im nordwestlichen Teil des Gemerské Rudohorie-Gebirges. Acta Fac. Rerum Nat. Univ. Comen., Bot. 26: 107-115.

MICHALKO J., BERTA J., MAGIC D. (eds). 1986. Geobotanická mapa ČSSR. Slovenská socialistická republika. Textová čast'. Veda, Bratislava. (in Slovak with German summary)

MIKYŠKKA R. 1929. Skalka v horách Štiavnických (Studie geobotanická). Věstn. Král. České Společn. Nauk. Tř. II, 1928: 1-23. (in Czech with French summary)

MIKYŠKA R. 1933. Vegetationsanalyse nebst einigen ökologischen Beobachtungen auf dem Berge Holík im Štiavnické středohoří (Schemnitzer Mittelgebirge). Beih. Bot. Cbl. 51: 354-373 .

MIKYŠKA R. 1939. Studie über die natürlichen Waldbestände im Slowakischen Mittelgebirge (Slovenské stredohorie). Ein Beitrag zur Soziologie der Karpatenwälder. Beih. Bot. Cbl. 59: 169-244.

MORAVEC J. 1985. Chorological and ecological phenomena in the differentiation and distribution of the Fagion associations in Bohemia and Moravia (Czechoslovakia). Vegetatio 59: 39-45 .

MORAVEC J. 1998. Přehled vegetace České republiky, Svazek 1. Acidofilní doubravy. Academia, Praha. (in Czech with English summary)

MORAVEC J. 1999. Differentiation of the Fagion and LuzuloFagion associations in the Czech Republic. Ann. Bot. 55(1997): 97-104.

MORAVEC J., HUSOVÁ M., CHYTRÝ M., NEUHÄUSLOVÁ Z. 2000. Přehled vegetace České republiky, Svazek 2. Hygrofilní, mezofilní a xerofilní opadavé lesy. Academia, Praha. (in Czech with English summary)

NEUHÄUSL R. 1977. Comparative ecological study of European oak-hornbeam forests. Natur. Canad. 104: 109-117.

NEUHÄUSL R., NEUHÄUSLOVÁ-NOVOTNÁ Z. 1964. Vegetationsverhältnisse am Südrand des Schemnitzer Gebirges. Biol. Práce 10(4): 1-80. 
NEUHÄUSLOVÁ-NOVOTNÁ Z. 1964. Zur Charakteristik der Carpinion-Gesellschaften in der Tschechoslowakei. Preslia 36: 38-54.

ONYSHCHENKO V.A. 2010. A revised classification of Ukrainian forests of the order Fagetalia sylvaticae. Tuexenia 30: 31-45.

PALLAS J. 1996. Beitrag zur Syntaxonomie und Nomenklatur der bodensauren Eichenmischwälder in Mitteleuropa. Phytocoenologia 26: 1-79.

POTT R. 1992. Die Pflanzengesellschaften Deutschlands. Eugen Ulmer Verlag, Stuttgart.

ROLEČEK J. 2005. Vegetation types of dry-mesic oak forests in Slovakia. Preslia 77: 241-261.

ROO-ZIELINSKA E., SOLON J. 1997. Effect of geographical location on species composition, vegetation structure, diversity and phytoindicative characteristics in pine forests. Environ. Pollut. 98: 347-360.

SLEZÁK M., KUKLA J. 2009. Forest vegetation of the northern part of the Štiavnické vrchy Mts. Folia Oecol. 36(1): 39-49.

SLEZÁK M., PETRÁŠOVÁ A. 2010. Oak forest vegetation in the northern part of the Štiavnické vrchy Mts (Central Slovakia). Hacquetia 9: 221-238.

ŠAMONIL P., VRŠKA T. 2008. Long-term vegetation dynamics in the Šmava Mts natural spruce-fir-beech forests. Plant Ecol. 196: 197-214.

ŠOMŠÁK L. 2000. Alnion glutinosae Malcuit 1929 na Slovensku (Západné Karpaty). Acta Fac. Rerum Nat. Univ. Comen., Bot. 40: 81-102. (in Slovak with English summary)

TER BRAAK C.J.F., ŠMILAUER P. 2002. CANOCO Reference manual and CanoDraw for Windows User's guide. Software for Canonical Community Ordination (version 4.5). Microcomputer Power, Ithaca-New York.

TICHÝ L. 2002. JUICE, software for vegetation classification. J. Veg. Sci. 13: 451-453.

TICHÝ L., CHYTRÝ M. 2006. Statistical determination of diagnostic species for site groups of unequal size. J. Veg. Sci. 17: 809-818.

TOWPASZ K., STACHURSKA-SWAKOŃ A. 2008. Alder-ash and willow communities and their diversity in the Pogórze Strzyzowskie Foothills (Western Carpathians). Acta Soc. Bot. Pol. 77: 327-338.

UJHÁZY K., HRIVNÁK R., BELANOVÁ E., BENČAŤOVÁ B. 2004. The beech forest vegetation of the Cerová vrchovina Mts (Southern Slovakia). Hacquetia 3: 61-73.

UJHÁZYOVÁ M. 2007. Syntaxonómia bukových lesov na vápencoch bradlového pásma. Technická univerzita, Zvolen. (in Slovak with English summary)

VON OHEIMB G., HÄRDTLE W. 2009. Selection harvest in temperate deciduous forests: impact on herb layer richness and composition. Biodivers. Conserv. 18: 271-287.

WILLNER W. 2002. Syntaxonomische Revision der südmitteleuropäischen Buchenwälder. Phytocoenologia 32: 337-453.

WILLNER W., GRABHERR G. 2007. Die Wälder und Gebüsche Österreichs. Ein Bestimmungswerk mit Tabellen. Spektrum Akademischer Verlag. Heidelberg.

WILLNER W., DI PIETRO R., BERGMEIER E. 2009. Phytogeographical evidence for post-glacial dispersal limitation of European beech forest species. Ecography 32: 1011-1018. 